\title{
Influência do Fósforo e de Diferentes Regimes de Corte na Produtividade e no Perfilhamento do Capim-de-Raiz (Chloris orthonoton Doell) ${ }^{1}$
}

\author{
Tatiana Neres de Oliveira ${ }^{2}$, Luiz Gonzaga da Paz ${ }^{3}$, Mércia Virginia Ferreira dos Santos ${ }^{4}$, José \\ Carlos Batista Dubeux Júnior ${ }^{4}$, Rinaldo Luiz Caraciolo Ferreira ${ }^{4}$, Aureliano José Vieira Pires ${ }^{5}$, \\ Maria da Conceição Silva ${ }^{2}$
}

\begin{abstract}
RESUMO - O trabalho foi conduzido em casa de vegetação do Departamento de Zootecnia/UFRPE, no período de outubro de 2000 a maio de 2001, objetivando avaliar o efeito da adubação fosfatada e de diferentes regimes de corte sobre a produtividade e perfilhamento do capim-de-raiz (Chloris orthonoton, Doell). O delineamento utilizado foi o inteiramente casualizado, distribuído em um arranjo fatorial $3 \times 2$ x 2, sendo três doses de adubação fosfatada $\left(0,100\right.$ e $200 \mathrm{~kg} /$ ha de $\left.\mathrm{P}_{2} \mathrm{O}_{5}\right)$, duas frequêencias de corte ( 30 e 40 dias) e duas intensidades de corte $(5$ e $15 \mathrm{~cm})$. A produção de MS foi influenciada pelas interações freqüência x intensidade de corte e adubação fosfatada x freqüência de corte. A melhor combinação entre freqüência e intensidade de corte foi de 40 dias e $5 \mathrm{~cm}$, propiciando produção de $10,66 \mathrm{~g} / \mathrm{vaso}$ de MS. A adubação fosfatada propiciou melhor resultado, quando a frequiência de corte foi de 40 dias (11,70 g/vaso de MS). O perfilhamento do capim-de-raiz foi influenciado pela adubação, freqüência e intensidade de corte.
\end{abstract}

Palavras-chave: adubação fosfatada, perfilhos axilares, perfilhos basais, produção de matéria seca

\section{Phosphorus and Cut System on the Productivity and Tillering of the Capim-de-raiz (Chloris orthonoton, Doell)}

\begin{abstract}
The work was carried out in a greenhouse, at the Animal Science Department/UFRPE, from October 2000 to May 2001, to evaluate the effect of phosphorus fertilization and different cut systems on the productivity and tillering of the capim-de-raiz (Chloris orthonoton, Doell). The experimental design was completely randomized, distributed in a factorial arrangement $3 \times 2 \times 2$, being three levels of application of phosphorus fertilizer $\left(0,100\right.$ and $200 \mathrm{~kg} / \mathrm{ha}$ of $\mathrm{P}_{2} \mathrm{O}_{5}$ ), two cut frequencies (30 and 40 days) and two cut intensities $(5$ and $15 \mathrm{~cm}$ ). Significant interaction between cut frequency and cut intensity as well as phosphorus fertilization and cut frequency for the DM yield was observed. The best combination between cut frequency and cut intensity was 40 days and $5 \mathrm{~cm}$, respectively, with $10.66 \mathrm{~g}$ /pot of DM. The phosphorus fertilization showed higher efficacy when the cut frequency was 40 days (11.70 $\mathrm{g}$ /pot of DM). Phosphorus fertilization, cut frequency and cut intensity showed significant effect on the tillering process.
\end{abstract}

Key Words: basal tillering, dry matter production, lateral tillering, phosphorus fertilizer

\section{Introdução}

A atividade pecuária no Brasil é voltada principalmente para os ruminantes e baseia-se no uso de pastagens nativas ou cultivadas para o suprimento de nutrientes para os animais. A baixa disponibilidade de $\mathrm{P}$ nos solos brasileiros acarreta grande prejuízo à produção das plantas, de modo geral, além de reduzir o perfilhamento e retardar o desenvolvimento das gramíneas forrageiras, fazendo com que a pastagem tenha uma cobertura deficiente, abrindo espaços para plantas invasoras (Rossi \& Monteiro, 1999).

O manejo de corte da forrageira é um fator que modifica tanto a produção quanto a qualidade da forragem. Cortes mais freqüentes resultam em menor produção de matéria seca (MS), porém de maior valor nutritivo que cortes menos freqüentes, que proporcionam produções mais elevadas de MS, porém de qualidade inferior (Alvim et al.., 2000).

Segundo Gerdes et al. (2000), a produção de forragem é um dos principais fatores capazes de afetar a produtividade de um sistema de pastejo. Corsi \& Santos (1995) definem o potencial de produção como a máxima produtividade biológica obtida sob condições ideais, sendo que a genética da planta forrageira define o potencial produtivo, enquanto $o$

\footnotetext{
1 Parte da Dissertação do primeiro autor apresentada ao Programa de Pós-Graduação em Zootecnia da UFRPE

2 Doutoranda do Programa de Pós-graduação em Zootecnia - UFRPE. E.mail: tneresdeoliveira@yahoo.com.br

3 Professor da UFRPE, Rua Manoel de Medeiros, s/no, Dois Irmãos, Recife-PE, CEP: 52171-900.

4 Professor da UFRPE, bolsista CNPq. E.mail: rmsantos@elogica.com.br; dubeux@edu.ufl

5 Professor Adjunto da UESB.
} 
manejo é responsável pela expressão dessa característica.

Souza et al. (1999a) relataram que um dos maiores problemas para o sucesso no estabelecimento e manutenção das espécies forrageiras é o nível extremamente baixo de $\mathrm{P}$ disponível. Além da sua deficiência natural em solos tropicais, a dinâmica de P no solo, principalmente as reações de adsorção aos óxidos de ferro e alumínio e precipitação com ferro e alumínio, que favorecem a sua imobilização química e a sua baixa mobilidade, contribuem para a necessidade de aplicação de altas doses de adubos fosfatados.

Existem poucas informações acerca do manejo do capim-de-raiz (Chloris orthonoton, Doell), uma gramínea nativa do Agreste pernambucano (Fernandes et al., 1983), apesar de representar parte da alimentação do rebanho no Semi-Árido.

Assim, o presente trabalho foi conduzido com o objetivo de avaliar a produtividade e o perfilhamento do capim-de-raiz sob diferentes níveis de adubação fosfatada, freqüências e intensidades de corte.

\section{Material e Métodos}

O trabalho foi realizado na casa de vegetação do Departamento de Zootecnia da Universidade Federal Rural de Pernambuco, na cidade do Recife, que se situa na microrregião fisiográfica do Litoral e Zona da Mata, no período de outubro de 2000 a maio de 2001. A cidade do Recife tem como coordenadas geográficas de posição: latitude $8^{\circ} 04^{\prime} 03^{\prime \prime} \mathrm{S}$; longitude $34^{\circ}$ 55' 00" W.Gr. e altitude de 4,0 m. O tipo climático é AMs, quente e úmido, e apresenta temperatura média anual de $25,2^{\circ} \mathrm{C}$ (FIDEPE, 1982).
O solo utilizado foi proveniente do Município de São Caetano-PE, local de ocorrência natural do capim-de-raiz (Chloris orthonoton, Doell). A análise de fertilidade das amostras do solo é apresentada na Tabela 1 e foi realizada no Laboratório de Fertilidade do Solo do Departamento de Agronomia da UFRPE.

Foram utilizados 48 vasos de plástico, medindo $30 \mathrm{~cm}$ de diâmetro e $22 \mathrm{~cm}$ de altura cada. Os vasos continham um orifício, onde foi introduzido um pedaço de mangueira de aproximadamente $10 \mathrm{~cm}$ de comprimento, constituindo o dreno. Para evitar o entupimento dos drenos pelo solo, foram colocados $2 \mathrm{~kg}$ de brita no fundo de cada vaso.

Foi realizada a calagem 60 dias antes do plantio, utilizando-se 1,0 t/ha de calcário dolomítico; em seguida, foi realizada a irrigação do solo para ativar a reação do calcário.

O plantio do capim-de-raiz foi realizado por meio de mudas enraizadas, provenientes de Caruaru-PE, no dia 11/12/2000, sendo utilizados quatro perfilhos/ vaso com pesos semelhantes e o corte de uniformização foi realizado 50 dias após o plantio, nas alturas correspondentes aos tratamentos.

As plantas foram irrigadas duas vezes por semana até a drenagem; a água drenada era reposta para os vasos para não haver perda de nutrientes por lixiviação.

Os tratamentos constaram da combinação de três doses de $\mathrm{P}_{2} \mathrm{O}_{5}\left(0,100\right.$ e $200 \mathrm{~kg} / \mathrm{ha}$ de $\left.\mathrm{P}_{2} \mathrm{O}_{5}\right)$, duas freqüências de corte ( 30 e 40 dias) e duas intensidades de corte (5 e $15 \mathrm{~cm}$ acima do solo), totalizando 12 tratamentos.

A adubação fosfatada, conforme tratamentos experimentais, foi realizada por ocasião do corte de uniformização e a fonte utilizada, o superfosfato

Tabela 1 - Análises química e física de amostra de solo de São Caetano-PE

Table 1 - Chemical and physical analysis of the soil sample from São Caetano-PE

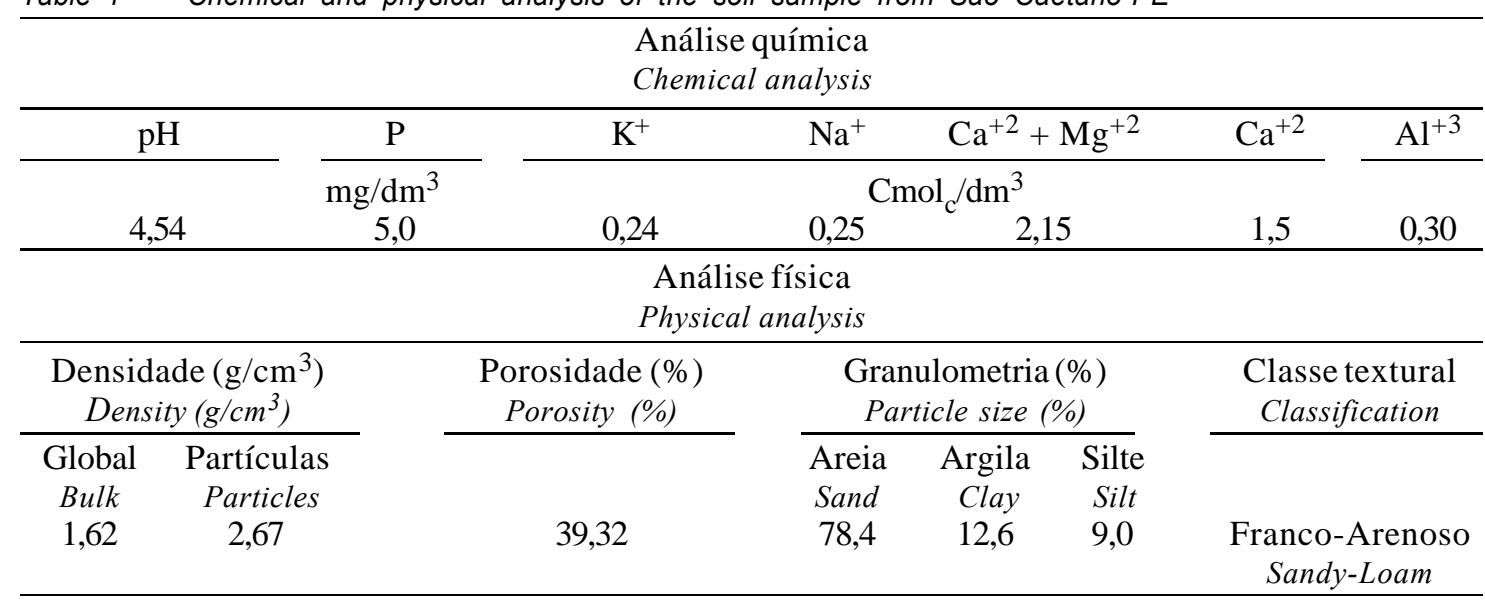


triplo, sendo que todos os tratamentos receberam a mesma quantidade de nitrogênio $(100 \mathrm{~kg} / \mathrm{ha}$ de $\mathrm{N}) \mathrm{e}$ potássio $\left(80 \mathrm{~kg} / \mathrm{ha}\right.$ de $\left.\mathrm{K}_{2} \mathrm{O}\right)$, utilizando-se como fontes dos nutrientes a uréia e o cloreto de potássio, respectivamente.

Os cálculos para se determinar a quantidade de cada nutriente/vaso foram feitos de acordo com a quantidade de solo utilizada/vaso $(12 \mathrm{~kg})$, densidade aparente do solo $\left(1,62 \mathrm{~g} / \mathrm{cm}^{3}\right)$ e profundidade efetiva das raízes $(20 \mathrm{~cm})$.

Foram realizados quatro cortes referentes ao tratamento de frequiência de corte de 30 dias e três cortes referentes ao de 40 dias. Por ocasião da colheita, amostras do material foram colocadas em sacos de papel, pesadas e colocadas em estufa de ar forçado $\left(65^{\circ} \mathrm{C}\right)$ por um período de 72 horas, para determinação dos teores de matéria pré-seca. Em seguida, foi realizada a determinação da matéria seca a $105^{\circ} \mathrm{C}$ (Silva, 1990) e os dados de produção transformados para matéria seca.

O número de perfilhos foi determinado pela contagem de perfilhos/vaso a cada colheita, sendo classificados em perfilhos basais e axilares, considerando o diâmetro de $30 \mathrm{~cm}$ dos vasos para efeito de cálculo na transformação de perfilhos $/ \mathrm{m}^{2}$, tendo o vaso área de $0,0706 \mathrm{~m}^{2}$.

A taxa de crescimento diário foi obtida pela relação entre a produção de MS e os dias de crescimento.

Devido à ocorrência de cochonilhas, as plantas foram submetidas a um tratamento com Confidor, cujo princípio ativo é a imida cloprid, durante o período experimental, utilizando-se $5 \mathrm{~g} / 10$ litros de água.

\section{Análises estatísticas}

O delineamento experimental utilizado foi o inteiramente casualizado, distribuído em um arranjo fatorial $3 \times 2 \times 2$, sendo três doses de $\mathrm{P}_{2} \mathrm{O}_{5}(0,100$ e $200 \mathrm{~kg} /$ ha de $\left.\mathrm{P}_{2} \mathrm{O}_{5}\right)$, duas intensidades de corte $(5$ e $15 \mathrm{~cm})$ e duas frequiências de corte ( 30 e 40 dias) com 12 tratamentos e quatro repetições, totalizando 48 parcelas experimentais. Foi utilizado o seguinte modelo estatístico:

$\mathrm{Y}_{\mathrm{ijkr}}=\mu+\mathrm{P}_{\mathrm{i}}+\mathrm{F}_{\mathrm{j}}+\mathrm{C}_{\mathrm{k}}+(\mathrm{PF})_{\mathrm{ij}}+(\mathrm{PC})_{\mathrm{ik}}+(\mathrm{FC})_{\mathrm{jk}}+$ $(\mathrm{PFC})_{\mathrm{ijk}}+$ Eijkr, com i $=1,2,3 ; \mathrm{j}=1,2 ; \mathrm{k}=1,2 \mathrm{e}$ $\mathrm{r}=1,2,3,4$

em que $\mathrm{Y}_{\mathrm{ijkr}}=$ valor observado da dose $\mathrm{i}$ de $\mathrm{P}$ combinada com os níveis j e $\mathrm{k}$ de $\mathrm{F}$ e $\mathrm{C}$ na repetição $\mathrm{r} ; \mu=$ média populacional geral; $\mathrm{P}_{\mathrm{i}}=$ efeito da dose $\mathrm{i}$ de adubação fosfatada; $F_{j}=$ efeito do nível $j$ de freqüência de corte; $\mathrm{C}_{\mathrm{k}}=$ efeito do nível $\mathrm{k}$ de intensidade de corte; $(\mathrm{PF})_{\mathrm{ij}}=$ interação entre a dose i de $\mathrm{P}$ e o nível j de F; $(\mathrm{PC})_{\mathrm{ik}}=$ interação entre a dose i de $\mathrm{P}$ e o nível $\mathrm{k}$ de $\mathrm{C} ;(\mathrm{FC})_{\mathrm{jk}}=$ interação entre o nível j de F e o nível $\mathrm{k}$ de C; $(\mathrm{PFC})_{\mathrm{ijk}}=$ interação entre a dose i de $\mathrm{P}, \mathrm{o}$ nível j de $\mathrm{F}$ e o nível $\mathrm{k}$ de $\mathrm{C} ; \mathrm{E}_{\mathrm{ijkr}}=$ efeito aleatório do erro experimental, conjuntamente independente de distribuição normal, média zero e variância comum $\sigma^{2}$.

\section{Resultados e Discussão}

Para produção de MS do capim-de-raiz, no primeiro corte, foi observado efeito significativo $(\mathrm{P}<0,05)$ para intensidade de corte e a interação $\mathrm{P}$ $\mathrm{x}$ frequiência de corte.

Entre as intensidades de corte (Tabela 3), houve diferença na produção de MS, sendo que o corte realizado a $5 \mathrm{~cm}$ de altura apresentou maior produção (7,30 g/vaso de MS), quando comparado com o corte a $15 \mathrm{~cm}$ de altura (6,70 g/vaso de MS). Estes resultados estão associados à maior proporção de material vegetal retirado em cortes mais baixos. Entretanto, vale ressaltar que cortes mais baixos e freqüentes podem comprometer a capacidade de rebrota das plantas.

A maior produção de MS obtida para o corte a $5 \mathrm{~cm}$ contraria afirmações de Maraschin (1995), de que cortes abaixo de $10 \mathrm{~cm}$ de altura promoveram rebrota menos vigorosa do capim Rhodes (Chloris gayana Kunth). Essa particularidade pode ser explicada pela diferença no hábito de crescimento entre as gramíneas, mesmo sendo do mesmo gênero. O capim-Rhodes (Chloris gayana Kunth) possui crescimento cespitoso e sua exigência em termos de fertilidade é relativamente alta, e sua rebrota pode ser comprometida em solos de fertilidade média ou baixa. O capimde-raiz (Chloris orthonoton Doell), apresenta crescimento estolonífero e é considerado como tolerante a solos de baixa fertilidade, já que sua ocorrência natural é em solos com essas características.

$\mathrm{Na}$ interação freqüência x $\mathrm{P}$ (Tabela 4), observa-se que no corte realizado aos 30 dias, as plantas que não receberam adubação fosfatada apresentaram menor produção (2,74 g/vaso de MS), quando comparadas às que receberam doses de 100 e $200 \mathrm{~kg} / \mathrm{ha}$ de $\mathrm{P}_{2} \mathrm{O}_{5}(6,90$ e 6,53 g/vaso de MS para 100 e $200 \mathrm{~kg} / \mathrm{ha}$ de $\mathrm{P}_{2} \mathrm{O}_{5}$, respectivamente), sendo estas semelhantes entre si. A mesma tendência foi observada no corte aos 40 dias de idade, em que a menor produção foi para as plantas não adubadas com $\mathrm{P}$ (5,16 g/vaso de MS) e a maior, para as adubadas com 100 e $200 \mathrm{~kg} / \mathrm{ha}$ de $\mathrm{P}_{2} \mathrm{O}_{5}(11,70$ e $11,70 \mathrm{~g} /$ vaso de MS para 100 e $200 \mathrm{~kg} / \mathrm{ha}$ de $\mathrm{P}_{2} \mathrm{O}_{5}$, 
respectivamente). Estes dados estão de acordo com aqueles encontrados por Gheri et al. (2000), que observaram aumento na produção de MS do capim Panicum maximum, Jacq. cv. Tanzânia com a aplicação de P. Na comparação doses de $\mathrm{P} \times \mathrm{FC}$, o corte aos 30 dias apresentou produção inferior nas três doses de $\mathrm{P}_{2} \mathrm{O}_{5}$ $(2,74 ; 6,90$ e $6,53 \mathrm{~g} /$ vaso de MS para 0,100 e $200 \mathrm{~kg} / \mathrm{ha}$ de $\mathrm{P}_{2} \mathrm{O}_{5}$, respectivamente), quando comparado com o corte aos 40 dias $(5,16 ; 11,70$ e 11,70 g/vaso de MS, respectivamente). Em geral, observa-se que, quando não foi adicionado $\mathrm{P}$ ao solo, a produção de forragem do capim-de-raiz foi limitada. Tais resultados estão de acordo com os observados por Souza et al. (1999b), onde as produções de MS de Brachiaria brizantha cv. Marandu aumentaram em função das doses de P apli- cadas. Gonzaga Neto et al. (1999), avaliando o efeito da adubação fosfatada no comportamento do capim-deraiz, confirmaram a resposta dessa gramínea à aplicação de $\mathrm{P}$ e o efeito desse elemento no crescimento vegetal das gramíneas.

No segundo corte, para a produção de MS houve efeito significativo $(\mathrm{P}<0,05)$ entre as doses de $\mathrm{P}$ e na interação freqüência $\mathrm{x}$ intensidade de corte.

As doses de P (Tabela 5) de 100 e $200 \mathrm{~kg} / \mathrm{ha}$ de $\mathrm{P}_{2} \mathrm{O}_{5}$ demonstraram produções superiores $(9,30 \mathrm{e}$ 9,12 g/vaso de MS, respectivamente), quando comparadas às produções das plantas que não foram submetidas à adubação fosfatada (3,96 g/vaso de MS). A semelhança na produção entre as doses de 100 e $200 \mathrm{~kg} / \mathrm{ha}$ de $\mathrm{P}_{2} \mathrm{O}_{5}$ demonstra que a produção

Tabela 2 - Resumo da análise de variância Table 2 - ANOVA summary

\begin{tabular}{|c|c|c|c|c|c|c|c|c|c|}
\hline \multirow[t]{2}{*}{$\begin{array}{l}\mathrm{FV} \\
S V\end{array}$} & \multirow[t]{3}{*}{$\begin{array}{l}\text { GL } \\
D F\end{array}$} & \multicolumn{5}{|c|}{$\begin{array}{c}\text { Teste } \mathrm{F} \\
F \text { test }\end{array}$} & \multirow[b]{2}{*}{$\begin{array}{c}\mathrm{TCD}^{2} \\
\text { (g MS/dia) } \\
D G R \\
\text { (g/day of } D M)\end{array}$} & \multirow[b]{2}{*}{$\begin{array}{r}\mathrm{PB}^{3} \\
B T\end{array}$} & \multirow[b]{2}{*}{$\begin{array}{c}\mathrm{PA}^{4} \\
A T\end{array}$} \\
\hline & & \multicolumn{4}{|c|}{$\begin{array}{c}\text { PMS (g/vaso })^{1} \\
D M Y(g / p o t)\end{array}$} & & & & \\
\hline & & $\begin{array}{c}10 \text { corte } \\
I^{s t} \mathrm{cut}\end{array}$ & $\begin{array}{l}2^{\mathrm{o}} \text { corte } \\
2^{\text {nd }} \mathrm{cut}\end{array}$ & $\begin{array}{c}3 \text { o corte } \\
3^{\text {rd }} \text { cut }\end{array}$ & $\begin{array}{c}4^{0} \text { corte } \\
4^{\text {th }} \mathrm{cut}\end{array}$ & & & & \\
\hline $\mathrm{P}$ & 2 & NS & $*$ & NS & NS & $\mathrm{T}$ & $*$ & $*$ & $*$ \\
\hline $\mathrm{FC}$ & 1 & NS & NS & $*$ & NS & & $*$ & NS & $*$ \\
\hline IC & 1 & $*$ & NS & NS & NS & & $*$ & NS & NS \\
\hline $\mathrm{P} \times \mathrm{FC}$ & 2 & $*$ & NS & NS & NS & & NS & NS & NS \\
\hline $\mathrm{P} \times \mathrm{IC}$ & 2 & NS & NS & NS & NS & & NS & $\mathrm{NS}$ & NS \\
\hline FC x IC & 1 & NS & $*$ & NS & NS & & NS & $\mathrm{NS}$ & NS \\
\hline $\mathrm{P} \times \mathrm{FC} \times \mathrm{IC}$ & 2 & NS & NS & NS & NS & & NS & NS & NS \\
\hline $\begin{array}{l}{ }^{*} \mathrm{P}<0,05 . \\
\mathrm{N} . \mathrm{S}: \mathrm{P} \geq 0,05 \\
1 \text { PMS: prod } \\
2 \text { TCD: taxa } \\
3 \text { PB: perfilh }\end{array}$ & & $\begin{array}{l}\text { ento d } \\
B T: b a\end{array}$ & $\begin{array}{l}\text { (DGR: d } \\
\text { ering). }\end{array}$ & $\begin{array}{l}\text { atter yie } \\
\text { growth }\end{array}$ & & & & & \\
\hline
\end{tabular}

Tabela 3 - Produção de matéria seca (PMS) do capim-de-raiz, conforme a intensidade de corte, primeiro corte

Table 3 - Dry matter yield (DMY) of capim-de-raiz, according to the cut intensity, first cut

\begin{tabular}{cc}
\hline $\begin{array}{c}\text { Intensidade de corte }(\mathrm{cm}) \\
\text { Cut intensity }(\mathrm{cm})\end{array}$ & $\begin{array}{c}\text { Produção de matéria seca }(\mathrm{g} / \mathrm{vaso}) \\
\text { Dry matter yield }(\mathrm{g} / \text { pot })\end{array}$ \\
\hline 5 & $7,30 \mathrm{a}$ \\
15 & $6,70 \mathrm{~b}$ \\
$\mathrm{CV}(\%)$ & 20,52
\end{tabular}

Médias seguidas de mesma letra não diferem estatisticamente pelo teste $\mathrm{F}$ $(0,05)$.

Means followed by the same letter are statistically similar $(P>0.05)$ according to $F$ test. 
não foi afetada pela maior dose aplicada, evidenciando que a utilização de níveis acima do recomendado podem não influenciar o crescimento da planta.

Em geral, observa-se que, quando não foi adicionado $\mathrm{P}$ ao solo, a produção de forragem do capim-deraiz (Chloris orthonoton Doell) foi limitada.

$\mathrm{Na}$ interação frequência $\mathrm{x}$ intensidade de corte (Tabela 6), observou-se que, quando cortadas aos 30 dias de idade, as plantas apresentaram produção de matéria seca semelhante nas duas alturas de corte $(5,77$ e 5,01 g/vaso de MS para 5 e $15 \mathrm{~cm}$, respectivamente). No corte realizado aos 40 dias, as plantas cortadas a $5 \mathrm{~cm}$ de altura apresentaram maior produção de MS (10,66 g/vaso de MS), quando comparadas ao corte de $15 \mathrm{~cm}(8,38 \mathrm{~g} /$ vaso de MS $)$. Na comparação IC x FC, considerando o corte realizado a $5 \mathrm{~cm}$ de altura, as maiores produções foram obtidas aos 40 dias de idade $(10,66 \mathrm{~g} /$ vaso de MS). O corte realizado aos $15 \mathrm{~cm}$ de altura apresentou a mesma tendência, sendo que a maior produção foi obtida aos 40 dias de idade ( $8,38 \mathrm{~g} / \mathrm{vaso}$ de MS), quando comparado com o corte aos 30 dias (5,01 g/vaso de MS).

Para a produção de MS do terceiro corte (Tabela 7), houve efeito significativo $(\mathrm{P}<0,05)$ apenas para freqüência de corte.

As plantas cortadas aos 40 dias de idade apresentaram maior produção de MS (9,85 g/vaso de MS), já aquelas cortadas aos 30 dias apresentaram menor produção $(5,39 \mathrm{~g} /$ vaso de MS). Esses resultados se

Tabela 4 - Produção de matéria seca (PMS) do capimde-raiz, conforme as doses de adubação fosfatada e frequência de corte, primeiro corte Table 4 - Dry matter yield (DMY) of capim-de-raiz, according to the phosphorus fertilization and cut frequency, first cut

\begin{tabular}{lccc}
\hline $\begin{array}{l}\text { Frequência de } \\
\text { corte (dias) } \\
\text { Cut frequency (days) }\end{array}$ & \multicolumn{3}{c}{$\mathrm{P}_{2} \mathrm{O}_{5}(\mathrm{~kg} / \mathrm{ha})$} \\
\cline { 2 - 4 } & \multicolumn{3}{c}{$\mathrm{PMS}(\mathrm{g} / \mathrm{vaso})$} \\
& \multicolumn{3}{c}{$D M Y(g /$ pot $)$} \\
30 & $2,74^{\mathrm{bB}}$ & $6,90^{\mathrm{aB}}$ & $6,53^{\mathrm{aB}}$ \\
40 & $5,16^{\mathrm{bA}}$ & $11,70^{\mathrm{aA}}$ & $11,70^{\mathrm{aA}}$ \\
$\mathrm{CV}(\%)$ & \multicolumn{3}{c}{16,42} \\
\hline
\end{tabular}

Médias seguidas de mesmas letras minúsculas na linha não diferem estatisticamente pelo teste Tukey $(P>0,05)$. Maiúsculas na coluna pelo teste $F(P>0,05)$.

Means followed by the same letter, lowercase letter in the row (Tukey test) and capital letter in the column ( $F$ test), are statistically similar $(P>05)$. justificam pelo estádio mais avançado das plantas cortadas aos 40 dias, conseqüentemente com maior crescimento vegetal e maior acúmulo de material vegetativo.

A taxa de crescimento diário pode ser observada na Tabela 8. Houve efeito significativo $(\mathrm{P}<0,05)$ para as doses de $\mathrm{P}$, freqüências e intensidades de corte (Tabela 8). O maior crescimento diário foi obtido nas plantas que receberam adubação fosfatada, sendo que nas doses de 100 e $200 \mathrm{~kg} / \mathrm{ha}$ de $\mathrm{P}_{2} \mathrm{O}_{5}$, os valores foram semelhantes $(0,25$ e $0,26 \mathrm{~g} / \mathrm{vaso} / \mathrm{dia}$ de $\mathrm{MS})$. As plantas que não receberam adubação fosfatada apresentaram as menores taxas de crescimento diário $(0,11 \mathrm{~g} / \mathrm{vaso} /$ dia de $\mathrm{MS})$. Com relação às freqüências e intensidades de corte, a maior taxa de crescimento foi observada nas plantas cortadas a $5 \mathrm{~cm}$ do solo $(0,23 \mathrm{~g} / \mathrm{vaso} / \mathrm{dia}$ de MS) e aos 40 dias de idade $(0,24 \mathrm{~g} / \mathrm{vaso} / \mathrm{dia}$ de MS). Isso está associado ao maior vigor de rebrota das plantas cortadas com mais intensidade e maior maturidade, onde, nestes casos, as maiores taxas de crescimento obtidas foram proporcionadas pelas plantas que apresentaram maior produção. $\mathrm{O}$ maior crescimento diário foi observado nas plantas que receberam adubação fosfatada, evidenciando a importância do $\mathrm{P}$ no crescimento de gramíneas, conforme observado por Luz et al. (2000).

Com relação ao número de perfilhos basais (Tabela 9), houve efeito significativo apenas para a adubação fosfatada $(\mathrm{P}<0,05)$. Não houve diferença significativa para número de perfilhos basais entre as doses de 100 e $200 \mathrm{~kg} / \mathrm{ha}$ de $\mathrm{P}_{2} \mathrm{O}_{5}$, sendo que as plantas que não receberam adubação fosfatada apresentaram valores inferiores aos demais.

Tabela 5 - Produção de matéria seca (PMS) do capimde-raiz, conforme a adubação fosfatada, segundo corte

Table 5 - Dry matter yield (DMY) of capim-de-raiz, according to the phosphorus fertilization, second cut

\begin{tabular}{lc}
\hline $\mathrm{P}_{2} \mathrm{O}_{5}(\mathrm{~kg} / \mathrm{ha})$ & $\begin{array}{c}\mathrm{PMS}(\mathrm{g} / \mathrm{vaso}) \\
D M Y(g / \text { pot })\end{array}$ \\
\hline 0 & $3,96^{\mathrm{b}}$ \\
100 & $9,30^{\mathrm{a}}$ \\
200 & $9,12^{\mathrm{a}}$ \\
$\mathrm{CV}(\%)$ & 38,06
\end{tabular}

Médias seguidas de mesma letra não diferem estatisticamente pelo teste Tukey $(P>0,05)$.

Means followed by the same letter statistically similar ( $P>.05)$, according to Tukey test. 
Tabela 6 - Produção de matéria seca (PMS) do capimde-raiz, conforme a frequência e intensidade de corte, segundo corte

Table 6 - Dry matter yield of capim-de-raiz, according to the cut frequency and cut intensity, second cut

\begin{tabular}{lcc}
\hline $\begin{array}{l}\text { Frequência de corte } \\
\text { Cut frequency }\end{array}$ & \multicolumn{2}{c}{$\begin{array}{c}\text { Intensidade de corte }(\mathrm{cm}) \\
\text { Cut intensity }(\mathrm{cm})\end{array}$} \\
\cline { 2 - 3 } & \multicolumn{3}{c}{$\begin{array}{c}\text { PMS (g/vaso) } \\
\text { DMY }(g / \text { pot })\end{array}$} \\
30 dias & $5,77^{\mathrm{aB}}$ & $5,01^{\mathrm{aB}}$ \\
30 days & & $8,38^{\mathrm{bA}}$ \\
40 dias & $10,66^{\mathrm{aA}}$ & \\
40 days & & \\
\hline $\mathrm{CV}(\%)$ & 16,42 \\
\hline
\end{tabular}

Médias seguidas de mesma letra não diferem estatisticamente pelo teste $F(P>0,05)$. Minúsculas na linha para a interação $F C x$ IC e maiúsculas na coluna na interação IC x FC.

Means followed by the same letter, lowercase letter at the row and capital letter at the column, are statistically similar (P>.05), according to $F$ test.
Tabela 7 - Produção de matéria seca (PMS) do capimde-raiz, conforme a freqüência de corte, terceiro corte

Table 7 - Dry matter yield (DMY) of capim-de-raiz, according to the cut frequency, third cut

\begin{tabular}{cc}
\hline $\begin{array}{c}\text { Freüência de } \\
\text { corte (dias) } \\
\text { Cut frequencyy (days) }\end{array}$ & $\begin{array}{c}\text { Produção de matéria } \\
\text { seca }(\mathrm{g} / \mathrm{vaso})\end{array}$ \\
\hline 30 & Dry matter yield $(\mathrm{g} /$ pot $)$ \\
\hline 40 & $5,39^{\mathrm{b}}$ \\
\hline $\mathrm{CV}(\%)$ & $21,85^{\mathrm{a}}$ \\
\hline
\end{tabular}

Médias seguidas de mesma letra não diferem estatisticamente pelo teste $\mathrm{F}(\mathrm{P}>0,05)$.

Means followed by the same letter statistically similar ( $P>.05)$, according to $F$ test.

Tabela 8 - Taxa de crescimento diário (TCD) do capim-de-raiz, conforme adubação fosfatada, freqüência e intensidade de corte

Table 8 - Daily growth rate (DGR) of the capim-de-raiz, according to the phosphorus fertilization, cut frequency and cut intensity

\begin{tabular}{|c|c|c|c|c|c|c|c|}
\hline & \multicolumn{3}{|c|}{$\mathrm{P}_{2} \mathrm{O}_{5}(\mathrm{~kg} / \mathrm{ha})$} & \multicolumn{2}{|c|}{$\begin{array}{l}\text { Frequência de } \\
\text { corte (dias) } \\
\text { Cut intensity } \\
\text { (days) }\end{array}$} & \multicolumn{2}{|c|}{$\begin{array}{l}\text { Intensidades } \\
\text { de corte }(\mathrm{cm}) \\
\text { Cut frequency } \\
(\mathrm{cm})\end{array}$} \\
\hline & 0 & 100 & 200 & 30 & 40 & 5 & 15 \\
\hline $\begin{array}{l}\text { TCD (g MS/dia }) \\
D G R(g D M / d a y)\end{array}$ & $0,11^{\mathrm{b}}$ & $0,25^{\mathrm{a}}$ & $0,26^{\mathrm{a}}$ & $0,18^{\mathrm{b}}$ & $0,24^{\mathrm{a}}$ & $0,23^{\mathrm{a}}$ & $0,18^{\mathrm{b}}$ \\
\hline $\mathrm{CV}(\%)$ & & & & 16,69 & & & \\
\hline
\end{tabular}

Médias seguidas de mesma letra não diferem $(P>0,05)$ entre si pelo teste Tukey para o $P$ e teste $F$ para FC e IC.

Means followed by the same letter are statistically similar $(P>0.05)$, according to Tukey test for phosphatic fertilization and $F$ test for cut frequency and cut intensity.

Fonseca et al. (1998) e Fonseca et al. (2000) encontraram resultados semelhantes, onde o número de perfilhos de Andropogon gayanus aumentou com as doses de $\mathrm{P}$, confirmando a relevância da adubação fosfatada sobre o processo de perfilhamento das gramíneas.

Já o número de perfilhos axilares (Tabela 10) foi influenciado pela adubação fosfatada, freqüências e intensidades de corte $(\mathrm{P}<0,05)$, não havendo efeito significativo $(\mathrm{P}>0,05)$ para as interações estudadas.

As plantas que não receberam adubação fosfatada apresentaram menor número de perfilhos axilares $\left(198,01\right.$ perfilhos $\left./ \mathrm{m}^{2}\right)$, sendo inferiores às plantas que receberam 100 e $200 \mathrm{~kg} / \mathrm{ha}$ de $\mathrm{P}_{2} \mathrm{O}_{5}(322,52$ e 310,33 perfilhos $/ \mathrm{m}^{2}$, respectivamente), os quais não diferiram entre si.
Entre as frequências de corte, o corte realizado aos 40 dias apresentou maior perfilhamento axilar (909,06 perfilhos $\left./ \mathrm{m}^{2}\right)$, quando comparado com o corte aos 30 dias $\left(654,95\right.$ perfilhos $\left./ \mathrm{m}^{2}\right)$. Este resultado pode estar associado ao fato de que cortes mais freqüentes resultam em plantas menores pela diminuição dos carboidratos de reserva armazenados pelas gramíneas na base do caule, limitando o desenvolvimento das plantas, como ressalta Rodrigues et al. (1987), e reduzindo o perfilhamento. Segundo Botrel et al. (1998), os menores intervalos de cortes refletem em menor peso das raízes, diminuindo o número de folhas e o perfilhamento das plantas.

Com relação aos perfilhos axilares, o corte realizado a $15 \mathrm{~cm}\left(877,33\right.$ perfilhos $\left./ \mathrm{m}^{2}\right)$ foi superior ao corte a $5 \mathrm{~cm}\left(685,26\right.$ perfilhos $\left./ \mathrm{m}^{2}\right)$. Estes resultados 
Tabela 9 - Número de perfilhos basais (perfilhos/área) do capim-de-raiz, conforme as doses de adubação fosfatada

Table 9 - Basal tiller number (tillers/area) of the capim-deraiz, according to the phosphorus fertilization levels

\begin{tabular}{lccc}
\hline & \multicolumn{3}{c}{$\mathrm{P}_{2} \mathrm{O}_{5}(\mathrm{~kg} / \mathrm{ha})$} \\
\cline { 2 - 4 } & 0 & 100 & 200 \\
\hline $\begin{array}{l}\text { Número de perfilhos } \\
\text { basais/m }\end{array}$ & $198,01^{\mathrm{b}}$ & $322,52^{\mathrm{a}}$ & $310,33^{\mathrm{a}}$ \\
Basal tiller number $/ \mathrm{m}^{2}$ & & & \\
$\mathrm{CV}(\%)$ & & 32,28 \\
\hline
\end{tabular}

Médias seguidas de mesma letra não diferem $(P>0,05)$ estatisticamente pelo teste Tukey.

Means followed by the same letter are statistically similar $(P>05)$, according to Tukey test. diferem daqueles encontrados por Coelho et al. (2001), os quais observaram que o aumento da intensidade de pastejo do Paspalum notatum resultou em aumento no perfilhamento, devido à redução da dominância apical. Santos et al. (2001) relataram que as gemas axilares do resíduo de capim-elefante "Roxo" apresentam intensas brotações, porém pouco vigorosas, não existindo um consenso quanto aos efeitos do desfolhamento sobre o surgimento de perfilhos durante o seu desenvolvimento vegetativo. O surgimento de maior número de perfilhos axilares, resultantes de cortes mais elevados, obedece a ordem natural da

Tabela 10 - Número de perfilhos axilares (perfilhos/área) do capim-de-raiz, conforme as doses de adubação fosfatada, frequências e intensidades de corte

Table 10 - Aerial tiller number (tiller/area) of the capim-de-raiz, according to the phosphorus fertilization level, cut frequency and cut intensity

\begin{tabular}{|c|c|c|c|c|c|c|c|}
\hline \multirow[b]{3}{*}{$\begin{array}{l}\text { Número de } \\
\text { Perfilhos } \\
\text { axilares } / \mathrm{m}^{2} \\
\text { Aerial tiller } \\
\text { number } / \mathrm{m}^{2}\end{array}$} & \multicolumn{3}{|c|}{$\mathrm{P}_{2} \mathrm{O}_{5}(\mathrm{~kg} / \mathrm{ha})$} & \multicolumn{2}{|c|}{$\begin{array}{l}\text { Frequência de } \\
\text { corte (dias) } \\
\text { Cut frequency } \\
\text { (days) }\end{array}$} & \multicolumn{2}{|c|}{$\begin{array}{c}\text { Intensidades de } \\
\text { corte }(\mathrm{cm}) \\
\text { Cut intensity } \\
(\mathrm{cm})\end{array}$} \\
\hline & 0 & 100 & 200 & 30 & 40 & 5 & 15 \\
\hline & $458,49^{b}$ & $888,81^{\mathrm{a}}$ & $972,09^{\mathrm{a}}$ & $654,95^{\mathrm{b}}$ & $909,06^{\mathrm{a}}$ & $685,26^{b}$ & $877,33^{a}$ \\
\hline $\mathrm{CV}(\%)$ & 49,70 & & & & & & \\
\hline
\end{tabular}

existência de maior número de gemas axilares resultantes de caules residuais.

Os resultados apresentados para perfilhamento, tanto basal quanto lateral, diferem daqueles encontrados por Gonzaga Neto et al. (1999), que não encontraram efeito significativo da adubação fosfatada no perfilhamento do capim-de-raiz cultivado em casa-de-vegetação.

Vale ressaltar a importância das informações geradas neste trabalho para auxiliar no direcionamento do manejo do capim-de-raiz no Semi-Árido, devido à existência do pequeno número de informações sobre a produção e o perfilhamento dessa gramínea nativa.

\section{Conclusões}

A adição de $\mathrm{P}$ e o corte realizado aos 40 dias proporcionaram maior produção de MS do capim-de-raiz.
As plantas adubadas com $\mathrm{P}$, a frequiência de corte de 40 dias e a altura de corte de $15 \mathrm{~cm}$ proporcionaram maior perfilhamento do capim-de-raiz.

\section{Literatura Citada}

ALVIM, M.J.; XAVIER, D.F.; VERNEQUE, R.S. et al. Resposta do tifton 68 a doses de nitrogênio e a intervalos de cortes. Pesquisa Agropecuária Brasileira, v.35, n.9, p.1875-1882, 2000.

BOTREL, M.A.; NOVAES, L.P.N.; ALVIM, M.J. Características forrageiras de algumas gramíneas tropicais. Juiz de Fora, MG: EMBRAPA-CNPGL, 1998. 35p. (Documentos, 66) COELHO, E.M.; HERLING, V.R.; GOMES, M.A. et al. Tillering dynamics in Mombaça grass (Panicum maximum, Jacq.) under grazing. In: INTERNATIONAL GRASSLAND CONGRESS, 19., 2001, Piracicaba. Proceedings ... Piracicaba: Fundação de Estudos Agrários Luiz de Queiroz, 2001. p.63.

CORSI, M.; SANTOS, P. M. Potencial de produção do Panicum maximum. In: SIMPÓSIO SOBRE MANEJO DA PASTA- 
GEM, 12., 1995, Piracicaba. Anais ... Piracicaba: Fundação de Estudos Agrários Luiz de Queiroz, 1995. p.275-303.

FERNANDES, A.P.M.; FARIAS, I.; LIRA, M.A. et al. Efeito de diferentes períodos de diferimento sobre o pasto de capim de Raiz (Chloris orthonoton Doell). In: CONGRESSO BRASILEIRO DE FORRAGEIRAS E PASTAGENS NATIVAS, 1., 1983, Olinda. Anais... IPA: Olinda, 1983. s.p.

FIDEPE - Informações municipais - Recife: FIDEM, 1982.

FONSECA, D.M.; GOMIDE, J.A.; ALVAREZ, V.H. Absorção, utilização, perfilhamento e níveis críticos de fósforo em gramíneas forrageiras. In: REUNIÃO ANUAL DA SOCIEDADE BRASILEIRA DE ZOOTECNIA, 35., 1998, Botucatu. Anais... Botucatu: Sociedade Brasileira de Zootecnia, 1998. p.312-314.

FONSECA, D.M.; GOMIDE, J.A.; ALVAREZ, V.H. et al. Absorção, utilização e níveis críticos internos de fósforo e perfilhamento em Andropogon gayanus e Panicum maximum. Revista Brasileira de Zootecnia, v.29, n.6, p.1918-1929, 2000.

GERDES, L.; WERNER, J.C.; COLOZZA, M.T. et al. Avaliação de características agronômicas e morfológicas das gramíneas forrageiras Marandu, Setária e Tanzânia aos 35 dias de crescimento nas estações do ano. Revista Brasileira de Zootecnia, v.29, n.4, p.947-954, 2000.

GHERI, E.O.; CRUZ, M.C.P.; FERREIRA, M.E. et al. Nível critico de fósforo no solo para Panicum maximum Jacq. cv. Tanzânia. Pesquisa Agropecuária Brasileira, v.35, n.9, p.1809-1816, 2000.

GONZAGA NETO, S.; TELES, M.M.; SANTOS, M.V.F. et al. Efeito da adubação no crescimento do capim-de-raiz (Chloris orthonoton Doell). In: REUNIÃO ANUAL DA SOCIEDADE BRASILEIRA DE ZOOTECNIA, 36., 1999, Porto Alegre. Anais... Porto Alegre: Sociedade Brasileira de Zootecnia, 1999. p.93-95.

LUZ, P.H.C.; HERLING, V.R.; LANG, A. et al. Efeitos de doses e fontes de fósforo na recuperação de Brachiaria decumbens Stapf. In: REUNIÃO ANUAL DA SOCIEDADE BRASILEIRA DE ZOOTECNIA, 37., 2000, Viçosa. Anais... São Paulo: SBZ/Gnosis, [2000]. CD-ROM. Forragicultura. FOR 0776.
MARASCHIN, G.E. Manejo de plantas forrageiras dos gêneros Digitaria, Cynodon e Chloris. In: SIMPÓSIO SOBRE MANEJO DA PASTAGEM, 9., 1995, Piracicaba. Anais... Piracicaba: Fundação de Estudos Agrários Luiz de Queiroz, 1995. p.88-99.

RODRIGUES, L.R.; MOTT, G.O.; VEIGA, J.B. et al. Effects of grazing management on leaf area and total nonstructural carbohydrates of dwarf elephantgrass. Pesquisa Agropecuária Brasileira, v.22, n.2, p.195-201, 1987.

ROSSI, C.; MONTEIRO, F.A. Doses de fósforo, épocas de coleta e o crescimento e diagnose nutricional nos capins Braquiária e colonião. Scientia Agrícola, v.56, n.4, p.11011110, 1999.

SANTOS, E.A.; SILVA, D.S.; QUEIROZ FILHO, J.L. Perfilhamento e algumas características morfológicas do Capim-elefante cv. Roxo sob quatro alturas de corte em duas épocas do ano. Revista Brasileira de Zootecnia, v.30, n.1, p.24-30, 2001.

SILVA, D.J. Análise de alimentos (métodos químicos e biológicos). 2.ed. Viçosa, MG: Universidade Federal de Viçosa, 1990. 165p.

SOUZA, R.F.; PINTO, J.C.; SIQUEIRA, J.O. et al. Micorriza e fósforo no crescimento de Brachiaria brizantha e Stylosanthes guianensis em solo de baixa fertilidade. 1. Produção de matéria seca e proteína bruta. Pasturas Tropicales, v.21, n.3, p.19-23, 1999a.

SOUZA, R.F.; PINTO, J.C.; SIQUEIRA, J.O. et al. Micorriza e fósforo no crescimento de Brachiaria brizantha e Stylosanthes guianensis em solo de baixa fertilidade. 1. Produção de matéria seca sob condições de estresse hídrico. Pasturas Tropicales, v.21, n.3, p. 31-35, 1999 b.

Recebido em: 05/07/02

Aceito em: 06/06/03 